\title{
A Smart Phone-based Personal Area Network for Remote Monitoring of Biosignals
}

\author{
M. J. Morón, J. R. Luque, A. A. Botella, E. J. Cuberos, E. Casilari and A. Diaz-Estrella \\ Dpto. Tecnología Electrónica, University of Málaga, Spain
}

\begin{abstract}
This paper presents a system whose purpose is to monitor a patient continuously from indoor or outdoor environments. The system is based on a Bluetooth PAN, carried by the patient, whose central node, a smart phone, compiles information about patient's location and health status. These data are encrypted to be sent to a server through Wifi or GPRS/UMTS. The system provides facilities to access to patient's data, even from a smart phone by a J2ME application. It also allows to configure remotely the threshold values used to detect emergency situations.
\end{abstract}

Keywords - Bluetooth, Smart Phone, pulsioximeter, PAN, J2ME.

\section{INTRODUCTION}

The increase in the processing and integration capacity of electronic devices, as well as the advances of low power wireless communications and, in general, wireless networking has enabled the development of unwired intelligent sensors for a wide set of applications. One of the most promising application fields is the medical telemonitoring of patients. Even a new concept: M-Health (or Mobile Health) has been proposed for the integration in a sanitary system of both mobile technologies and wireless sensors [1]. The generic goal of an M-health system is to increase the patient mobility and allow the enable the sanitary agents to access the medical information seamlessly and with independence of the physical location of both (patient and sanitary staff).

The incorporation of mobile technologies to the medical care has diverse benefits: mobile solutions have been shown to help improve patient safety, decrease the risk of medical errors and increase physician productivity and efficiency. Similarly, wireless sensors enable the patients' freedom of movements and promote new ways of patient monitoring such as home monitoring [2]. This clearly improves patients' quality of life. Missed days of school or work are reduced and health related restrictions on normal daily activities are minimized. Moreover, it allows the "service continuity" of the healthcare attention, as it defines a constant link to medical professionals who are able to assist in the disease management process. Patient telemonitoring facilitates the extension of healthcare services to remote and sparse-populated areas avoiding the need of expensive medical premises. On the other hand, providing physicians and clinicians wireless access to patient information and medical references largely eliminates the need to locate and read through patient charts or search for lab results from other departments. Telemonitoring also increments the medical presence in emergency scenarios and makes possible remote diagnosis.

As a consequence of the advances in wireless technologies, the architecture of a classical telemedicine service has strongly evolved [3]. Initial telemetry systems just contemplated the simple retransmission of the biosignals captured by wired sensors via a POTS modem or ISDN. Today new networking technologies as well as new communication paradigms (such as 'Context Aware' or 'Always Best Connected') enable new possibilities in telemonitoring services, ranging from limited indoor ('short distance') scenarios (e.g.: a care units) to outdoor applications without any mobility restrictions.

The paradigm of present (and future) medical monitoring systems is the definition of a W-PAN (Wireless Personal Area Networks) or W-BAN (Wireless Body Area Networks). The PAN (or BAN) must integrate a set of wearable wireless devices capable of sensing and transmitting biosignals. In most cases, the PAN/BAN is coordinated by a node which in turn may retransmit the signals to a remote central monitoring unit [4]. Thus, the general architecture of a PAN/BAN considers three components [2]:

- A medical sensor network (which can be completed with movement and positioning sensors), equipped with a low power, short range wireless interface, mainly based on Bluetooth or ZigBee technologies.

- A coordinator central node that communicates with sensors and, simultaneously, acts as an Internet gateway to other networks (GSM/GPRS/UMTS or WLAN) in order to transmit sensors biosignals (or medical alarms) and to receive control information.

- A central node (or a distributed central system) in charge of storing the sensors signals, detecting possible alarms and distributing the patients' information (e.g: via Internet, SMS, e-mail, etc) to the medical staff.

Optionally, the medical staff may receive the patients' information in a handheld (PDA, phone) specifically designed or programmed for this purpose. 
This article presents an architecture that defines a monitoring network of Bluetooth biosensors connected to a commercial 3G cell phone with a WLAN interface. The main goal of this architecture is to evaluate in the future the performance of smart phones when used as the gateway/master in a PAN of Bluetooth sensors. The evaluation especially will take into consideration the limitations of the extended J2ME (Java Mobile Edition) as a designing tool for this type of monitoring applications.

The rest of the work is structured as follows: Section 2 describes related works. Section 3 summarizes the specifications of the architecture and briefly describes the general structure of the prototype. Section 4 comments in more detail the implementation. Finally section 5 presents some conclusions and project's current status.

\section{RELATED WORKS ON WIRELESS PAN}

The first projects that introduced the concept of Personal Area networks (PAN) [6][7] employed proprietary systems for wireless transmissions. The work in [6], for example, described a PAN that integrated the information generated by different intelligent sensors. The wireless interface of the PAN utilised a $916 \mathrm{MHZ}$ RF transceptor from Link Technologies which provided a bi-directional bit-rate of 33.6 Kbps in a range of $50 \mathrm{~m}$. Similarly, in [7] authors develop a PAN system which interconnects low-power sensors to PDAs and PCs by means a radio transceptor that operates at 916 or $433 \mathrm{MHz}$ with a bit rate of $76.8 \mathrm{kbps}$ and a coverage radio of $20-30 \mathrm{~m}$.

In spite of these initial works, the present tendency in the field of medical PANs (and telemedicine in general) is the utilisation of standards for the different wireless communications that the PAN requires. The use of standards notably reduces the development cost while easing the deployment of telemedicine systems and product interoperability [8].

In case of requiring to reduce as much as possible the sensor consumption (which is basic aspect when dealing with implanted sensors whose batteries cannot be easily replaced), Zigbee/802.15.4 based transmission may be the best choice (see, for example, the works in [2] or [9]). However, in applications where battery restrictions are not so exigent, Bluetooth (BT) is by far the most utilised technology by designers to interconnect PAN sensors with the monitoring system unit (and or signal gateway). Among the main reasons that make this popular standard an attractive candidate to dynamically monitor physiological parameters the literature usually mentions the small size, reduced cost and low power consumption of the BT radio modules, the BT technique of frequency hopping (which increases secu- rity and privacy in radio transmissions) [4] [10], the capability of BT to conform scatternets and ad hoc networks, the potentiality of BT for wearable systems [11][8][12], and specially the present penetration of BT in the market (and its related commercial support) when designing solutions for short range transmission. Additionally the bandwidth supported by BT (up to $1 \mathrm{Mb} / \mathrm{s}$ ) is enough to convey (and still multiplex) any real time biosignal.

Diverse prototypes of BT sensors have been developed for different biosignals, including ECG, glucometers, tensiometers, pulse-oximeters and even stethoscopes [10]. In fact, during last three years several vendors (Corscience, Nonin Medical, A\&D Medical, etc.) have launched numerous homologated wireless biosensors with Bluetooth interfaces which can be easily integrated in a Body Area Network. The apparition of these commercial Bluetooth terminals permits a straightforward integration of general purpose devices (PDAs, embedded PCs, Mobile Phones,...) in the design of the PAN/BAN networks (which is the main goal of this work). So, the system can benefit from the computing power of these devices just by simply programming Bluetooth communications through conventional programming libraries (e.g. BlueZ). This increases the versatility and capability of reconfiguration of the network, reducing its deployment time and its final cost.

Different examples of networking architectures have been proposed in the literature to solve the problem of medical telemonitoring.

The AMON Project [13] has implemented a portable equipment in the wrist of the patient. This equipment is capable of measuring several biosignals simultaneously (SPO2, ECG, blood pressure, the temperature and the patient movements). The developed equipment processes the signals and, in case of medical alert, communicates with the medical center by means of the cellular network (sending a SMS or creating a connection of switched circuit through GSM). However, as it is difficult to integrate multiple biosensors in a single device, today most medical telemetry systems include some kind of 'wearable piconet' of independent wireless sensors. In most cases, the architecture consists of a (wearable or not) 'multiplexing' PAN node which collects the biosignals from one or more BT (or wired) biosensors. Once the signals are received, the node directly shows them on an embedded display or (more commonly) it retransmits them to a central node (normally located on medical premises) by means of a medium (Wifi) or long range (GPRS, UMTS) transmission technology.

For example, in the works [14] and [15] an ECG signal is transmitted via BT to a PALM device and a smart phone, respectively. These devices in turn forward the signal to a 
server through GPRS. A similar system for ECG monitoring, presented in [16], adds to the GPRS transmitted flow the compressed information from a GPS. Analogously, the architecture in [4] allows a wearable unit (PDA or cell phone) to receive via BT the signals from a ECG but also from a pulse monitor and a tensiometer. In [17] a pulsioximeter and a tensiometer are integrated in the same device which transmits the signals to mobile phone via BT. A J2ME programmed application in the phone allows to process the signals and detect medical alarms. In that case, a SMS is sent to the medical staff. If the mobile service is not operative, the sensor (which can remotely configured) is able to store the signals temporally until GPRS service is recovered. The architecture in [18] also includes a mobile wearable equipment which act as gateway between the BT sensors and a GPRS/UMTS connected central unit. The main particularity of the system is the utilisation of XML for the configuration of the sensors.

Authors in [5] develop a piconet of BT smart sensors. The sensors can be programmed by a control node which is remotely accessed by GPRS/UMTS. The same authors present in [19] another piconet of wearable sensors. The control node is situated in a cell phone or PDA with Wifi, GPRS and UMTS interfaces.

A similar system is described in [3]. In this case, the piconet is substituted by a set of wired sensors connected to a microprocessor board specifically designed for this application. The board also includes a BT module to intercommunicate with the hospital through a GPRS mobile phone. In the reception part medical staff can receive the information of the patients in portable PDAs.

In contrast with previous works (in which BT communications are programmed at HCI or L2CAP layers), the main novelty of the BT piconet described in [20] is that, in order to ease the interoperability among different vendors, BT sensors are accessed by using Bluetooth Serial Port Profile.

The work in [21] introduces a PAN architecture comprising a set of plug and play BT sensors (including ECG, pulsioximeter, humidity and temperature sensors) controlled by a wearable data logger. By using BT the logger connects to an Internet-attached base station which stores the signals in a distributed data base. The interoperability of the sensors is achieved by means of the ISO/IEEE 11073 (Medical Information Bus) standard.

Authors in [22] remark that the main goal of a telemonitoring system is to transmit eventual medical alarms. As these systems are aimed at patients and elder people who spend most time at their homes, the work proposes the use of not-wearable (fixed) BT access points that will be re- sponsible for collecting the signals and transmitting them to a central unit (a PDA) in a hospital.

From these experiences, we can remark the growing use of 'general purpose' mobile devices (mainly PDAs or Cell Phones) to build the PAN node which acts as the gateway between the wireless sensors and the final remote control node. This can be justified by the increasing computing power, storing and communicating capabilities (BT is included in an important percentage of models) as well as by the decreasing costs of these devices. Moreover, due to the universalised use of cell phones in developed countries, the implementation of the medical BAN central node can be performed without introducing any new wearable hardware and just with a re-configuration of a familiar and quotidian element in the everyday life of the patients (the mobile phone).

As it refers to the programming language that is normally chosen to build the software in the phones (or PDAs), initial architectures utilised $\mathrm{C}$ or $\mathrm{C}++$, which permitted an optimised design for real time processing and a better interaction with underlying operative system (e.g: Symbian in the case of most phones). However, since the apparition of J2ME (Java version for mobile devices) and in spite of its limitations (for example, with the APIs to incorporate BT), the portability of Java is notably stimulating its adoption in the field of medical BANs.

\section{SySTEM DESCRIPTION}

The profile of the target user is a patient who needs continuous monitoring. The patient usually resides in his own home or in a shared environment with other patients or users, i.e. a residence for elder people. This scenario will be referred as a "controlled environment". Besides, health status is not an obstacle to leave the controlled environment to go for a walk, for example. In this case the patient will be in "outdoor scenario". The purpose is to carry out a smart tracking of patients to achieve the following advantages:

- To avoid patients to have to stay on bed and connected by cable to a medical monitor

- To increase the patient's mobility and comfort level.

- To allow remote monitoring in outdoor spaces.

- To reduce number of periodical check-up.

\section{A. Functions}

It is intended to provide the next functions:

1. Monitoring parameters related to health status in order to detect an emergency. 
2. Recording of the outdoor location of patient.

3. To provide medical assistance in case of emergency.

The wireless transmission of patient's information will be protected by means of authentication and encryption.

\section{B. Architecture}

Regardless the patient's environment, there are three basic parts or components in the system:

1. An iBAN worn by the patient to be remotely monitored.

2. A Central Control Server (CCS), located in the controlled environment.

3. A Mobile Control and Monitoring Unit (MCMU) carried by the physicians.

The iBAN prototype is based on Bluetooth technology. Currently the iBAN comprises a commercial medical sensor, a pulsioximeter, a GPS device and an Intelligent Node, referred as IN. The IN integrates two wireless communications interfaces, 802.11 and Bluetooth, as well as a connection to cellular network. Bluetooth is the technology used for communications between the devices and the IN, which plays the master role in the piconet. Wifi and/or GPRS/UMTS are employed to send information from the IN to the CCS and to the MCMU.

In the following subsections, the interaction between the different components is described.

\section{Patient iBAN-CCS Interaction}

An application, which resides in the IN, manages the Bluetooth piconet and the CCS connections. When it is automatically started, i.e., without user request, the next sequence of steps is carried out: (1) inquiry procedure, (2) services search, (3) connection establishment with pulsioximeter and GPS devices, (4) pulsioximeter configuration and (5) server connection and user registration.

As IN receives data from iBAN nodes, it stores them during a period which can be remotely configured. When the time is out, the IN sends these data to the server by the 802.11 interface in case that the user is in a controlled environment and there is an available Wifi access point. In other case, the data will be transmitted by GPRS/UMTS.

As in [5], the Bluetooth connections are kept active as long as the devices are in the coverage area of the IN. Just as the authors of [5] point out, the advantage is that, as soon as an event occurs, the IN can notify it to the server. However, the main drawback of this policy is the increase in power consumption.

The data rate depends on the mode operation of the iBAN:
- Default Mode (mode 1): The pulsioximeter sends 3 bytes/s. The information from GPS is also received and stored in the IN, but it is not retransmited to the CCS.

- Verbose Mode (mode 2): This operation mode has to be remotely enabled from the server or the MCMU. In this case the pulsioximeter sends a rate of 375 bytes/s. SPO2 data and GPS parameters are delivered to the CCS.

Furthermore, if the verbose mode is activated and the GPS device does not provide valid data, the latest stored parameters are recovered.

Additionally, in both modes, the information received from the pulsioximeter is processed in order to verify that the oxygen saturation and the heart rate are not out of the security range established for the patient. If the IN detects a value lower or greater than a predefined threshold, a SMS will be sent to the physician mobile, and, if desired, to some patient's relatives. The thresholds can be set up by a physician from the server or his own MCMU.

Furthermore, periodically the CCS requests IN to switch to verbose mode in order to check battery status. This is necessary because the chosen commercial pulsioximeter only includes this indicator under this mode.

The sequence diagram displayed in figure 1 shows the initialization procedure, the default mode operation and the enabling of the verbose mode.

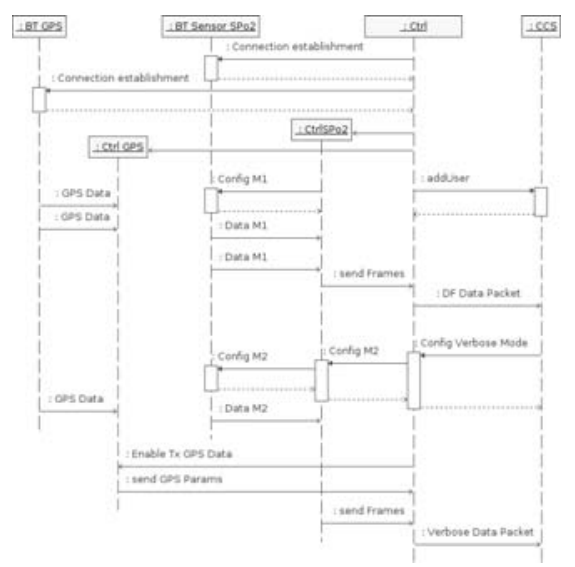

Fig. 1 Diagram of the interaction between the iBAN sensors and the CCS

\section{MCMU-CCS Interaction}

When a MCMU receives an alert SMS from a patient, the physicians starts a J2ME control and monitoring application. This one allows to select a patient and the sensor whose data the physician wants to display. In order to get requested data, a midlet establishes a TCP connection with the CCS. Through this connection, the CCS retransmits the 
frames received from iBAN in quasi-real time. The transmission is not stopped until the physician selects the corresponding option.

Additionally, the application provides the possibility of configuring the operation mode, the thresholds values used to detect an emergency or even the transmission period. In order to guarantee the reliability these configuration commands are sent through a TCP connection.

\section{IMPLEMENTATION}

The next devices has been incorporated in the iBAN prototype: (1) a smart phone, initially a Nokia 9500 model and later, a Nokia N93, as hardware platforms for the intelligent node (IN), (2) a Bluetooth pulsioximeter, from Nonin Medical [23]. This sensor implements SPP profile, and a Bluetooth GPS receiver with SirfStarIII chipset and SPP profile too.

For the Mobile Control and Monitoring Unit (MCMU) a smart phone has been also employed.

\section{A. IN and $M C M U$}

A J2ME application has been implemented for the IN. The first version, developed for the Nokia 9500 (Series 80 2nd.), uses the next APIs: Bluetooth API (JSR-82) for managing BT connections and Wireless Messaging API (JSR120) for sending SMSs.

The midlet, in a second version implemented for the Nokia $\mathrm{N} 93$ (Series $603 \mathrm{rd}$. Edition), extends the functionality by using: (1) Security and Trust Services API (JSR-177) to include data encryption; (2) the Location API (JSR-179) to get data from GPS with more reliability. The last version of Wireless Messaging API (JSR-205) has been used instead of JSR-120. Additionally, the File Connection API (JSR75) has been employed to record patient's information in files that can be created into the file system residing on the mobile device or on an external memory card.

The need of privacy when transmitting and processing medical data is the main reason to select the model Nokia N93. To encrypt data in the transmission to CCS, a symmetric algorithm DES with a prefixed key, previously arranged between both communication end points, is utilised.

The control and monitoring application for the MCMU also has been developed as a midlet. The program also employs Security and Trust Services API (JSR-177).

The screenshot displayed in fig. 2 shows pulsioximeter's parameters during an execution in Nokia N93.

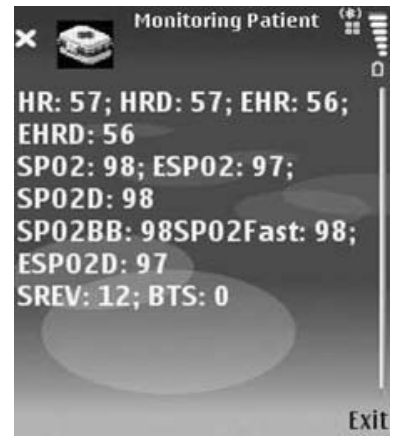

Fig.2. Screenshot of monitoring midlet

\section{B. $C C S$}

The CCS is an Apache web server implemented as two servlets under Tomcat. The first one carries out two main functions: (1) it manages the connection requests from patients to be monitored, compiling and processing medical data (2) it provides access to the IN capabilities by a web interface using applets. The second servlet serves access requests from the MCMUs carried by physicians and retransmit data from the IN to MCMU through a TCP connection.

The communication between CSS and patient's iBAN can be established using two protocols: (a) HTTP: data are sent in the HTTP request itself. If the server has to send a command, it will use a HTTP message. (b) TCP-UDP/IP: commands requested to the IN as well as the responses to commands and indications are transmitted through TCP, whereas the data are sent over UDP.

In relation to provide an easy access to physicians, two mechanisms are included:

1. Web interface: The physician can access the system through a PC, from the hospital or in any location with Internet access, using a navigator with RMI support, such as Firefox, by means of a JApplet.

This JApplet is structured in two main components: (a) Configuration Interface, to manage the iBAN configuration and operation mode, and (b) Display Data, to show health parameters and location information. The Configuration Intereface component communicates with CSS by The RMI. Display Data subsystem also uses RMI to get, in real-time, the parameters extracted from decoded frames in the server.

2. Mobile Network: The control and monitoring midlet installed on the MCMU. The physician only has to start the midlet, which will establish a TCP connection with the server to receive the encrypted frames from the IN which were redirected by the CSS. 


\section{Conclusions}

The use of smart phones as central node in a PAN to compile information about patient's location and health status, is favourable because it takes advantage of the user's acquaintance with mobile device. However, the main restriction from usability perspective is that the monitoring application carries out tasks considered by operating system as risky for security. Therefore acknowledgement is requested to user before continuing certain actions. Nevertheless, this drawback could be avoided by means of a validated certificate, instead of the self certificate used.

On the other hand, the J2ME implementation for monitoring applications is advantageous since its portability is greater than Symbian. Nonetheless, it has been detected that the problems occurred in the midlet implementation depend on the smart phone. The phone Nokia 9500 generates Symbian errors during the SPP connection establishment. It has been checked that these errors also happen with other applications that use the SPP profile. However, the SPP connection establishment works successfully in Nokia N93 and Nokia N70 (Series 60 2nd. Edition FP3). However, only in Nokia N93, sometimes, the midlet freezes during Wifi access point searching. Symbian errors also take place in Nokia 9500 when a Bluetooth connection with a device is dropped and the application tries to recover it.

Finally, it has been verified with Nokia N93 that the application continues running when the cover is closed, on the contrary to other experiences [18].

\section{ACKNOWLEDGMENTS}

This work has been supported by Projects No. TIC200307953-C02-01 and TEC2006-12211-C02-01/TCM.

\section{REFERENCES}

1. Istepanian R S, Jovanov E, Zhang Y T (2004) Introduction to the Special Section on M-Health: Beyond Seamless Mobility and Global Wireless Health-Care Connectivity, IEEE Trans. on Inf. Tech. in Biomedicine, vol. 8, pp 405-414, Dec. 2004.

2. Jovanov E, Milenkovic A, Otto C et al. (2005) A wireless body area network of intelligent motion sensors for computer assisted physical rehabilitation, Jour. of Neuroengineering Rehabilitation, vol. 2, no. 6.

3. Rasid A, Woodward B (2005) Bluetooth Telemedicine Processor for Multichannel Biomedical Signal Transmission via Mobile Cellular Networks, IEEE Trans.on Inf. Tech. in Biomedicine, vol. 9, pp 35-43.

4. Hung K, Zhang, Y, Tai B (2004) Wearable medical devices for telehome healthcare, in Proc. of Conf. EMBC 2004, vol. 7, pp 5384 5387.

5. Krco S (2003) Implementation solutions and issues in building a personal sensor network for health care monitoring, in Inf. Tech. Applications in Biomedicine, 2003. 4th International IEEE EMBS Special Topic Conference on, pp 350-353.
6. Jovanov E, Price J, Raskovic D et al. (2000) Wireless Personal Area Networks in Telemedical Environment, in Proc. of the IEEE EMBS Intern. Conf. on Inf. Tech. Applications in Biomedicine, pp 22-27.

7. Malan A D, Fulford-Jones T, Welsh M (2004) CodeBlue: An Ad Hoc Sensor Network Infraestructure for Emergency Medical Care, in Proc. of MobiSys WAMES 2004, Boston, MA, pp 12-14.

8. Warren S, Yao J, Schmitz R et al. (2003) Wearable telemonitoring systems designed with interoperability in mind, in Proc. of IEEE Eng. in Medicine and Biology Society, 2003, vol. 4, pp 3736-3739.

9. Timmons N, Scanlon W (2004) Analysis of the performance of IEEE 802.15.4 for medical sensor body area networking, in Proc. of IEEE SECON 2004, pp 16-24.

10. Hung K, Zhang Y (2002) Usage of Bluetooth in wireless sensors for tele-healthcare, in [Engineering in Medicine and Biology, 2002. 24th Annual Conference and the Annual Fall Meeting of the Biomedical Engineering Society] EMBS/BMES Conference, 2002. Proc. of the Second Joint, vol. 3, pp 1881-1882.

11. Warren S, Yao J, Schmitz R et al. (2004) Reconfigurable Point-ofCare Systems Designed with Interoperability Standards, in Proc. of EMBC 2004, vol. 2, pp 3270-3273.

12. Yao J, Schmitz R, Warren S (2005) A wearable point-of-care system for home use that incorporates plug-and-play and wireless standards, Information Technology in Biomedicine, IEEE Trans. on, vol.9, pp 363-371.

13. Anliker U, Ward J, Lukowicz P et al. (2004) AMON: a wearable multiparameter medical monitoring and alert system, IEEE Trans.on Inf. Tech. in Biomedicine, vol. 8, pp 415 - 427.

14. Dong J, Zhu H (2004) Mobile ECG detector through GPRS/Internet, in Computer-Based Medical Systems, 2004. CBMS 2004. Proc.. 17th IEEE Symposium on, pp 485- 489.

15. Khoor S, Nieberl K, Fugedi K et al. (2001) Telemedicine ECGtelemetry with Bluetooth technology, Computers in Cardiology 2001, pp 585-588.

16. Liszka K, Mackin M, Lichter (2004) Keeping a beat on the heart, in Pervasive Computing, IEEE, vol. 3, pp 42-49.

17. Lee R G, Hsiao C C, Chen C C et al. (2006) A mobile-care system integrated with bluetooth blood pressure and pulse monitor, and cellular phone, IEICE Trans. on Information and Systems, vol. E89-D, pp 1702-1711.

18. Krco S, Kostic S, Sakac D et al. (2005) mSens mobile health monitoring system, vol. 1, pp 80-83, The International Conference on Computer as a Tool. EUROCON 2005.

19. Krco S, Delic V (2003) Personal wireless sensor network for mobile health care monitoring, in Proc. of TELSIKS 2003, vol. 2, pp 471474.

20. Wang D, Lu Y, Zhang $\mathrm{H}$ et al. (2005) A wireless sensor network based on Bluetooth for telemedicine monitoring system, in Microwave, Antenna, Propagation and EMC Technologies for Wireless Communications, vol. 2, pp 1361- 1364.

21. Warren S, Lebak J, Yao J et al. (2005) Interoperability and Security in Wireless Body Area Network Infrastructures, in Engineering in Medicine and Biology Society, 2005. IEEE-EMBS 2005. 27th Annual International Conference of the, pp 3837-3840.

22. Park D, Kang S (2004) Development of reusable and expandable communication for wearable medical sensor network, in Proc. of. EMBC 2004, vol. 7, pp 5380- 5383.

23. Nonin Medical Inc. at http://www.nonin.com 